\title{
Raconter ensemble. L'exemple de l'épisode de la rencontre avec la chatte dans Danger dans le potager
}

Tell a story together or not. The example of the meeting episode with the she-cat in Danger in the garden

\section{Marianne Drusch}

\section{(2)enEdition}

\section{Journals}

Édition électronique

URL : http://journals.openedition.org/pratiques/3996

DOI : $10.4000 /$ pratiques.3996

ISSN : 2425-2042

Éditeur

Centre de recherche sur les médiations (CREM)

\section{Référence électronique}

Marianne Drusch, «Raconter ensemble. L'exemple de l'épisode de la rencontre avec la chatte dans Danger dans le potager », Pratiques [En ligne], 177-178 | 2018, mis en ligne le 20 juillet 2018, consulté le 10 décembre 2020. URL : http://journals.openedition.org/pratiques/3996 ; DOI : https://doi.org/ 10.4000/pratiques.3996

Ce document a été généré automatiquement le 10 décembre 2020.

(c) Tous droits réservés 


\section{Raconter ensemble. L'exemple de l'épisode de la rencontre avec la chatte dans Danger dans le potager}

Tell a story together or not. The example of the meeting episode with the she-cat in Danger in the garden

\section{Marianne Drusch}

1 La maitrise de la langue orale est la finalité première à l'école maternelle. L'une des activités phares dans les classes concerne la restitution de récit en groupe : il s'agit pour l'enseignant d'obtenir de ses élèves qu'ils rappellent les évènements marquants d'une narration tout en rendant cette restitution la plus cohérente possible. La cohérence du récit est assurée par la gestion de deux plans qui le structurent (Fayol, 1994).

En situation de groupe, quand les élèves sont amenés à raconter à plusieurs une histoire connue de tous, l'enseignant peut individualiser les échanges et selon le type d'étayage qu'il apporte à l'enfant obtenir des résultats plus ou moins proches de ce que l'on aurait en situation de production individuelle. Le danger bien connu étant que pour soutenir la performance il est amené à multiplier les interventions avec comme conséquence pédagogique une perte d'attention progressive d'une partie du groupe.

Ce constat est confirmé dans le rapport de l'Inspection générale consacré à «La pédagogie de l'oral : toujours autant de faiblesses et les mêmes difficultés » (MEN, 2011) :

«En règle générale, dans les classes, il est indéniable qu'il y a du langage, mais le volume et le temps de parole des enfants sont parfois peu élevés, surtout dans les écoles qui accueillent massivement des élèves de milieux défavorisés. [...] De fait, il se trouve presque toujours quelques élèves qui parlent, même si l'on constate aussi le cas d'élèves silencieux, peu toniques. Et ceux qui parlent font avancer l'échange ; le maitre suit alors son fil directeur, attend les bonnes réponses, voire les suggère [...]. Souvent, c'est par une grêle de questions magistrales que le langage des enfants est suscité ».

Les séances de langage se caractérisent donc par une prise de parole très inégale entre élèves, avec une incitation forte des enseignants qui favorise quelques-uns d'entre eux. 
Dans le cadre d'une activité de rappel collectif de récit, le questionnement structurant auquel se tiennent la plupart des enseignants semble être le moyen naturel de le faire restituer. L'enseignant utilise ce que V. Rivière (2006: 249) nomme des «consignes réactives " pour répondre à ses attentes, notamment lorsqu'il souhaite que ses élèves passent d'une idée à une autre, enchainent deux évènements dans une narration : « Les consignes réactives exercent une contrainte (plus) forte sur la prise de parole suivante (force illocutoire et pragmatique plus directe) et revêtent un caractère obligatoire ».

5 Ce «dialogue pédagogique ordinaire» (Nonnon, 1997) a deux conséquences majeures. Tout d'abord, il laisse trop peu de place à l'initiative des élèves. On peut rappeler les observations souvent citées de M. Joulain (1990:64) sur une dizaine de séances de langage en maternelle. L'auteure constate que « les séances de langage [qu'elle observe] s'organisent sur le mode préférentiel d'échanges individuels courts (entre la maitresse et un enfant particulier) ; $70 \%$ d'entre eux en moyenne ne dépassent pas 2 tours, soit un aller-retour entre la maitresse et un enfant particulier ». Ensuite, il ne favorise pas l'écoute puisque c'est l'enseignant qui reste maitre d'œuvre des échanges sans qu'il y ait d'autonomie dans la prise de parole des élèves.

On le sait, l'enseignant peut aussi adopter une posture en retrait et favoriser l'écoute entre les élèves (Péroz, 2010). Ce faisant, ceux-ci ont la possibilité de reprendre très naturellement des éléments déjà fournis par d'autres élèves mais qu'ils n'auraient pas nécessairement mémorisés. De même, ils peuvent compléter un énoncé en rappelant un détail qui aurait pu être oublié initialement ou revenir sur le sens d'une information en développant un élément implicite. Enfin, certaines informations apportées isolément peuvent être rassemblées par un lien syntaxique. Plusieurs modes de reprises ou de reformulations sont donc envisageables.

7 Est-il alors possible de sortir du paradoxe du «dialogue pédagogique ordinaire » et permettre aux élèves de travailler à l'apprentissage d'une conduite discursive essentielle, la narration, en adoptant une autre posture pédagogique? Les élèves peuvent-ils développer un épisode sans être dirigés de manière précise par l'enseignant?

En posant que le travail collaboratif lié au retrait de l'enseignant a des effets sur la restitution, nous formulons deux hypothèses: la première est que le rapport au texte initial est transformé, ce qui a pour résultat d'obtenir mieux qu'un squelette évènementiel, la seconde est que le collectif à travers les reprises-reformulations est la source principale de ces améliorations.

9 Pour vérifier ces hypothèses, nous étudierons la restitution d'un épisode complexe de Danger dans le potager sur les 12 séances du corpus CLEA. Cette étude s'articulera autour des questions suivantes:

- Quels sont les éléments du texte qui sont restitués?

- Les éléments restitués ont-ils des caractéristiques communes? Autrement dit, la nature des informations a-t-elle une influence sur leur mémorisation/restitution?

- Comment les éléments sont-ils restitués? Quels sont les processus linguistiques et langagiers que les élèves utilisent?

- Quels sont les effets de la gestion de groupe? Quelles sont les variations produites selon le type de retrait pratiqué par l'enseignant?

10 Nous présenterons d'abord les variables de situation en proposant une analyse du textesource, fondateur de la cohérence narrative, en déterminant la nature des informations de la trame narrative et les relations entre ces informations. Dans un deuxième temps, 
nous décrirons la méthodologie adoptée pour délimiter la partie du corpus concernée et déterminer les critères d'analyse. Ensuite, nous exposerons les résultats en nous focalisant sur les informations restituées, la prise en charge collective des informations, et l'analyse de deux séances particulières.

\section{Le texte-source}

Le texte Danger dans le potager rapporte une action unifiée, tendue vers un but général. Pierre lapin veut échapper à son poursuivant, Monsieur Grégory et retrouver son foyer. Trois évènements liés à trois lieux différents ponctuent la fuite : les vêtements de Pierre lapin qui gênent sa course dans le potager, l'arrosoir dans la cabane qui se révèle être une mauvaise cachette et la rencontre à la mare. Nous avons sélectionné ce dernier épisode que l'on va appeler l'épisode de la chatte blanche parce qu'il est paradoxalement le moins narratif des trois. Nous verrons tour à tour la spécificité de cet épisode, les informations qu'il comporte et les relations entre ces dernières.

\section{La spécificité de l'épisode}

Le corpus étudié se réfère à la restitution de ce dernier épisode, dont le thème est la rencontre à la mare.

«La course folle reprend de plus belle; il cherche désespérément à sortir du potager. Il arrive près d'une mare, où une chatte blanche observe un poisson rouge dont elle ferait bien son repas. Sa queue frétille d'impatience et Pierre n'ose pas la déranger, ne sachant pas si, comme le lui a dit son cousin, les chats sont vraiment les ennemis des lapins! »

Dans les deux épisodes précédents du jardin et de la remise, Pierre est confronté directement à Monsieur Grégory. Dans ce troisième épisode, il est mis en présence d'un nouveau personnage, la chatte, personnage secondaire dans l'histoire, qu'il ne connait pas et qui pourrait constituer une menace. Il s'agit donc d'une situation contradictoire: s'il avance et se fait repérer par la chatte, il risque de se faire attaquer et s'il attend trop longtemps ou s'il revient en arrière, il risque de se faire attraper par son pire ennemi. Pour résoudre ce nouveau problème, Pierre s'appuie sur une recommandation de son cousin et fait le choix de ne pas déranger la chatte. Pierre prend ainsi une sage décision qui lui permet de se sortir de cette situation délicate et de rejoindre sans encombre son domicile. Contrairement aux autres épisodes de la fuite, la réaction de Pierre tient moins dans ses capacités physiques, être rapide et habile, que dans la qualité de sa réflexion et de sa prise de décision.

Deux éléments semblent donc distinguer ce troisième épisode: la présence d'un personnage secondaire, dont Pierre ne sait pas s'il est réellement menaçant, et la réflexion de Pierre qui précède sa réaction. L'étape à la mare apparait dans la fuite du héros comme un non-évènement, une péripétie avortée.

Cet épisode se caractérise également par sa brièveté, ce qui présente un avantage dans le traitement du corpus. 


\section{Les informations dans la trame narrative}

16 Cet épisode, même s'il n'est qu'une phase de la course poursuite parmi d'autres, possède une dynamique propre, générée par un début - Pierre rencontre le chat - et progressant vers une fin - Pierre continue son chemin. C'est le dernier épisode du potager.

\section{Les informations}

Les séquences verbales qui constituent le texte de cet épisode donnent au lecteur les informations qui constituent le fil de l'histoire. Selon leur statut dans la chronologie des évènements elles se situent au premier ou au deuxième plan du récit et jouent un rôle distinct dans la chronologie (importance de l'information).

\section{Les plans}

18 À la suite des travaux de J.-M. Mandler et N. S. Johnson (1984), la plupart des auteurs différencient les informations du premier plan de celles du second plan. Les premières font avancer la chronologie des évènements relatifs au personnage principal, les secondes renseignent sur les conditions objectives du monde dans lequel évoluent les protagonistes du récit ou sur leurs émotions et leurs états d'esprit. L'emploi du présent qui peut dire à la fois les évènements du premier plan et ceux du second (commentaires, descriptions, explications, etc.) ne permet pas de clarifier cette opposition des plans qui constitue la structure temporelle du récit (Hickmann, 2000).

19 Pierre est le personnage principal de l'épisode retenu, c'est sa participation aux évènements qui fait de ceux-ci qu'ils appartiennent au premier plan, lequel constitue le fil de l'histoire. Les informations relatives à la chatte sont du deuxième plan tout comme ce qui relève des états internes de Pierre, à l'exception de la décision de ne pas déranger la chatte. C'est cette information qui déclenche en effet le départ du jeune lapin, et signifie qu'il continue la course.

20 Le tableau ci-dessous regroupe l'ensemble des séquences verbales de l'épisode, associées aux informations correspondantes. Les informations sont numérotées (deuxième colonne) et sont classées selon qu'elles relèvent du premier plan (en jaune) ou du deuxième plan (en bleu). La dernière colonne indique s'il s'agit d'informations principales ou secondaires. 
Tableau 1. Répartition des informations selon les séquences verbales

\begin{tabular}{|l|l|l|l|l|}
\hline Séquence verbale & No & $\begin{array}{l}\text { Information } \\
\text { de premier plan }\end{array}$ & Information de deuxième plan & $\begin{array}{l}\text { Importance de } \\
\text { l'information }\end{array}$ \\
\hline La course folle reprend de plus belle & 1 & $\begin{array}{l}\text { Pierre court, et court } \\
\text { encore plus vite }\end{array}$ & $\begin{array}{l}\text { Information } \\
\text { principale }\end{array}$ \\
\hline $\begin{array}{l}\text { Il cherche désespérément à sortir du } \\
\text { potager }\end{array}$ & 2 & & Pierre veut sortir du potager. & $\begin{array}{l}\text { Information } \\
\text { secondaire }\end{array}$ \\
\hline Il arrive près d'une mare & 3 & Pierre arrive à la mare. & & $\begin{array}{l}\text { Information } \\
\text { principale }\end{array}$ \\
\hline $\begin{array}{l}\text { Où une chatte blanche observe un } \\
\text { poisson rouge }\end{array}$ & 4 & & Il y a une chatte blanche. & $\begin{array}{l}\text { Information } \\
\text { principale }\end{array}$ \\
\hline & 5 & & Il y a un poisson rouge. & $\begin{array}{l}\text { Information } \\
\text { secondaire }\end{array}$ \\
\hline Dont elle ferait bien son repas. & 7 & & La chatte observe un poisson. & $\begin{array}{l}\text { Information } \\
\text { secondaire }\end{array}$ \\
\hline Sa queue frétille d'impatience & 8 & & La chatte veut manger le poisson. & $\begin{array}{l}\text { Information } \\
\text { secondaire }\end{array}$ \\
\hline Et Pierre n'ose pas la déranger & 9 & $\begin{array}{l}\text { Pierre ne dérange pas la } \\
\text { chatte. }\end{array}$ & Sa queue frétille. & $\begin{array}{l}\text { Information } \\
\text { secondaire }\end{array}$ \\
\hline $\begin{array}{l}\text { Ne sachant pas si les chats sont les } \\
\text { ennemis des lapins }\end{array}$ & 10 & & $\begin{array}{l}\text { Pierre a un doute... il ne sait pas si } \\
\text { les chats sont les ennemis des } \\
\text { lapins. }\end{array}$ & $\begin{array}{l}\text { Information } \\
\text { principale }\end{array}$ \\
\hline principale \\
\hline
\end{tabular}

\section{L'importance des informations}

21 L'importance des informations dans le déroulement de l'histoire ne se confond pas exactement avec la hiérarchie des plans (Barthes, 1966). Certes, les informations de premier plan correspondent à des informations principales, par contre les informations de deuxième plan peuvent logiquement être des informations secondaires (informations $2,5,5,7,8,11$ ) mais celles qui jouent un rôle essentiel dans le déclenchement des évènements de premier plan sont des informations principales. Le texte en présente deux : la présence de la chatte et le doute de Pierre qui ne sait pas si les chats sont les ennemis des lapins (informations 4 et 10).

Il y a cinq informations principales : Pierre court, il arrive près d'une mare et y rencontre un obstacle potentiel qui est la chatte. Pierre ne la dérange pas car elle peut être son ennemie.

Il y a six informations secondaires : Pierre cherche encore à sortir du jardin. Son cousin l'a mis en garde contre les chats. Une chatte observe un poisson rouge pour le manger tout en frétillant de la queue. Ces informations secondaires se rapportent aux conditions objectives du monde, à l'état interne de la chatte, et à un évènement passé. Elles contribuent au principe de cohérence textuelle.

24 Nous allons à présent examiner comment les informations sont reliées dans le texte même.

\section{Les relations entre les informations}

L'épisode de la mare est inclus dans une séquence narrative plus large, la fuite de Pierre, dont le but qui structure son action est d'échapper à Monsieur Grégory. L'épisode rappelle le but du personnage qui apporte de nouveaux évènements et de nouvelles actions qui s'enchainent de manières différentes selon leur rapport à ce but. Voyons comment se font les enchainements. 
Selon les travaux de J.-M. Mandler et N. S. Johnson (1984), les informations sont reliées entre elles par trois types de relation: la simultanéité lorsque deux informations sont simultanées ou se chevauchent temporellement, la succession lorsqu'elles se succèdent dans le temps, et la causalité dès lors que la première information fournit une raison à l'occurrence de la seconde. Cette dernière relation relie deux informations de manière plus étroite que les relations temporelles de simultanéité ou de succession. C'est elle qui assure la séquentialité des informations.

Tableau 2. Relations entre les informations

\begin{tabular}{|c|c|c|c|}
\hline Séquence verbale & Information & $\mathrm{N}^{\mathbf{0}}$ & Relation des informations \\
\hline La course folle reprend de plus belle & Pierre court et court plus vite encore. & 1 & \\
\hline $\begin{array}{l}\text { Il cherche désespérément à sortir du } \\
\text { potager }\end{array}$ & Pierre veut sortir du potager. & 2 & Simultanéité entre 1 et 2. \\
\hline Il arrive près d'une mare & Pierre arrive à la mare. & 3 & Succession entre 2 et 3 . \\
\hline \multirow[t]{3}{*}{$\begin{array}{l}\text { Où une chatte blanche observe un } \\
\text { poisson rouge }\end{array}$} & Il y a une chatte blanche. & 4 & Simultanéité entre 3 et 4. \\
\hline & Il y a un poisson rouge. & 5 & Simultanéité entre 4 et 5 . \\
\hline & La chatte observe le poisson. & 6 & Simultanéité entre 5 et 6. \\
\hline Dont elle ferait bien son repas. & La chatte veut manger le poisson. & 7 & Cause entre 6 et 7 \\
\hline Sa queue frétille d'impatience & Sa queue frétille. & 8 & Cause entre 8 et 3 . \\
\hline Et Pierre n'ose pas la déranger & Pierre ne dérange pas la chatte. & 9 & Succession entre $2,[4,5,6]$ et 9 . \\
\hline $\begin{array}{l}\text { Ne sachant pas si les chats sont vraiment } \\
\text { les ennemis des lapins. }\end{array}$ & $\begin{array}{l}\text { Pierre a un doute... il ne sait pas si les chats sont les } \\
\text { ennemis des lapins. }\end{array}$ & 10 & Cause entre 9 et 10. \\
\hline Comme le lui a dit son cousin & Le cousin de Pierre a donné une information. & 11 & Cause entre 10 et 11. \\
\hline
\end{tabular}

D'un point de vue temporel, quatre informations sont reliées par des relations de simultanéité et deux sont reliées par des relations de succession.

Cet épisode est peu évènementiel, puisqu'il ne comporte que deux relations de succession: Pierre arrive près de la mare et décide de ne pas déranger la chatte. Il est donc difficile à restituer, comme le rappelle l'étude de H. Makdissi et A. Boisclair (2004) qui identifie plusieurs niveaux de compétences pour les enfants de 3 ans $1 / 2$ à 6 ans $1 / 2$ dans la restitution d'un texte après une lecture préalable par l'adulte ${ }^{1}$ : les enfants produisent au mieux un squelette d'actions, actions qui s'enchainent d'abord sur le plan temporel puis ensuite sur le plan de la causalité.

Les relations de causalité, au nombre de quatre, sont plus nombreuses et présentent des informations enchâssées et donc dépendantes. Elles ne sont pas marquées explicitement par des connecteurs linguistiques, mais sont établies en tenant compte de la relation sémantique entre les informations, de sorte que l'une peut être considérée comme la raison ou la finalité de l'autre. Selon E. Veneziano et C. Hudelot (2005), nous distinguons également deux formes de causalité. Dans un premier cas, la causalité peut suivre le déroulement temporel : il s'agit alors d'un mouvement dit "proactif». Ainsi, la chatte observe le poisson (information 6) parce qu'elle veut le manger (information 7). Dans un second cas, le sens du déroulement temporel des évènements est contraire à celui présenté par la relation: la queue de la chatte frétille (information 8) parce qu'elle veut manger le poisson (information 7).

30 Le texte-source présente donc des caractéristiques particulières pour cet épisode, tant dans sa compréhension que dans sa mémorisation et sa restitution. La première porte sur l'effet de la suspension de l'action principale qui est la fuite et ce, en raison de la passivité $\mathrm{du}$ personnage principal et $\mathrm{du}$ caractère secondaire des actions attribuables au personnage de la chatte. La seconde est le nombre important des relations de causalité entre les informations. Ces relations de causalité renvoient à des savoirs du monde (la queue de la chatte frétille d'impatience parce qu'elle aimerait manger le poisson) mais aussi à un état interne qui explique le comportement de Pierre (il ne dérange pas la chatte car il n'est 
pas sûr que les chats soient les ennemis des lapins). On constate également que ces relations ne sont pas marquées par des connecteurs explicites dans le texte (parce que, donc, alors...). Le rôle de connecteur est en particulier joué par le participe présent ne sachant pas.

\section{Le traitement des données}

31 Les interventions des élèves sont elles-mêmes tributaires du texte-source qu'il s'agit de restituer. Les restitutions s'organisent toujours à partir de données textuelles initiales, des éléments constitutifs du récit.

Après avoir montré la place de la restitution de cet épisode dans le corpus, nous présenterons la méthodologie de notre analyse, basée sur le séquençage des énoncés des élèves, pour recenser les informations restituées et les identifier en fonction de leur importance.

\section{La place de la restitution de l'épisode dans le corpus}

L'épisode de la mare est la troisième péripétie de Danger dans le potager qui en comporte deux autres qui ont lieu respectivement dans le jardin et dans la remise. Les péripéties les plus marquées (relations temporelles essentiellement) sont celles qui sont les plus citées par les enfants, comme le mentionnent H. Makdissi et A. Boisclair (2004). C'est bien le cas ici, puisque l'épisode qui nous intéresse est le moins rappelé, comme le montre le graphique ci-dessous.

Figure 1. Restitution des péripéties

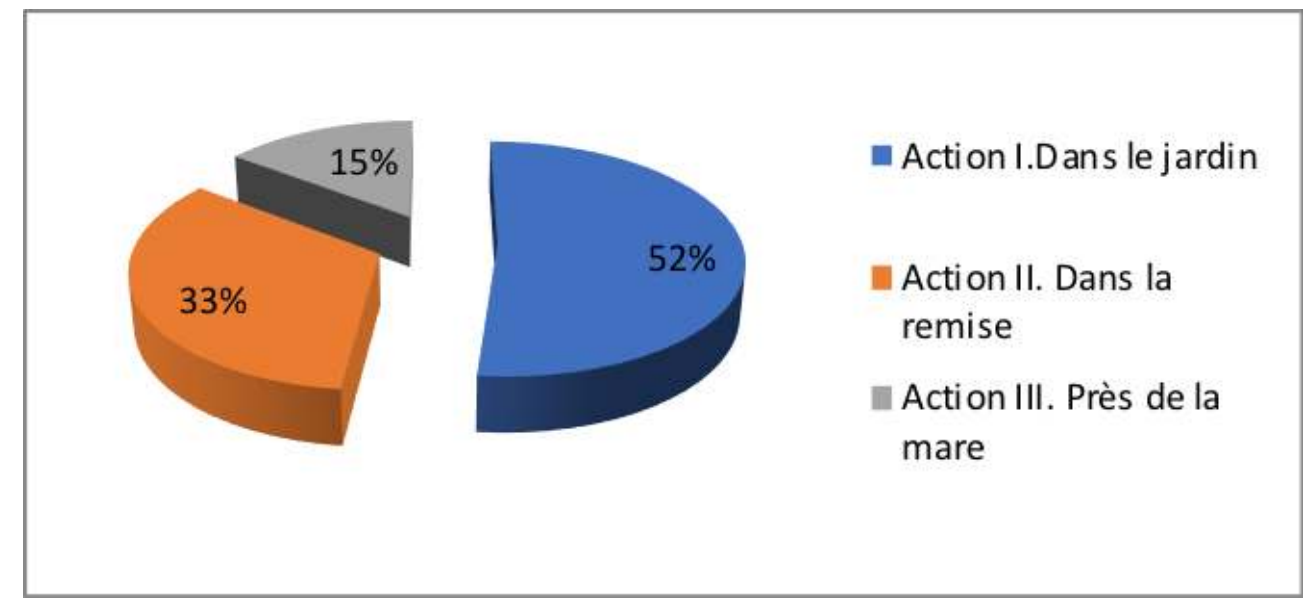

Ce constat est conforté par le faible nombre d'interventions par séance restituant l'épisode de la mare. Le corpus regroupe seulement 71 interventions réparties sur les 12 séances, et distribuées de la façon suivante.

Tableau 3. Restitution de l'épisode sur l'ensemble des séances

\begin{tabular}{|l|l|l|l|l|l|l|l|l|l|l|l|l|l|}
\hline séance & 1 & 2 & 3 & 4 & 5 & 6 & 7 & 8 & 9 & 10 & 11 & 12 & total \\
\hline $\begin{array}{l}\text { Nombre d'interventions } \\
\text { portant sur l'épisode }\end{array}$ & 3 & 3 & 21 & 9 & 4 & 7 & 1 & 3 & 9 & 4 & 3 & 4 & $\mathbf{7 1}$ \\
\hline $\begin{array}{l}\text { Nombre total } \\
\text { d'interventions } \\
\text { d'enfants dans le corpus }\end{array}$ & 57 & 209 & 322 & 170 & 74 & 89 & 82 & 110 & 101 & 98 & 95 & 88 & $\mathbf{1 4 9 5}$ \\
\hline $\begin{array}{l}\text { \% d'interventions par } \\
\text { séance }\end{array}$ & $5,3 \%$ & $1,4 \%$ & $6,5 \%$ & $5,3 \%$ & $5,4 \%$ & $7,9 \%$ & $1,2 \%$ & $2,7 \%$ & $8,9 \%$ & $4,1 \%$ & $3,2 \%$ & $4,5 \%$ & $4,75 \%$ \\
\hline
\end{tabular}




\section{Le mode d'analyse} interventions peut comporter une ou plusieurs informations parmi les 11 relatives à l'épisode de la mare. Elles sont identifiées au regard de la segmentation proposée des informations. En effet, les enfants ne produisent pas un écrit individuel mais interagissent en situation d'oral collectif. Les prises de parole dépendent de ce qui s'est dit précédemment et sont insérées dans ce mouvement discursif. Avant leur début, il y a les énoncés des autres, et ensuite il y a les énoncés-réponses à venir (François, 1989). Ainsi, un enfant peut mémoriser et restituer une information qui, à son tour, peut être reprise, complétée, transformée par un autre enfant, alors même que celui-ci ne l'avait pas d'abord mémorisée lui-même.

manière générale, le sens des énoncés est déterminé par l'alternance des interventions : un enfant peut compléter l'énoncé d'autrui par un syntagme nominal ou, dans une situation de concurrence dans la prise de parole, donner le plus économiquement possible une information nominale avec ou sans présentatif. En situation d'oral collectif, ces tours de parole qui pourraient être considérés comme "déficitaires» au regard de la norme scripturale, doivent être considérés comme apportant aussi des informations, aussi éloignées soient-ils de la forme écrite initiale.

Nous considérons donc que les formes non verbales tout comme les énoncés introduits par un présentatif, même s'ils ne sont pas suivis d'une proposition, constituent déjà une information.

Tableau 4. Exemples d'informations nominales avec ou sans présentatif

\begin{tabular}{|l|l|l|l|}
\hline Séances & $\mathbf{N}^{\mathbf{0}}$ & Loc & Interventions \\
\hline 3 & 250 & Clarissa & une chatte blanche/ une chatte blanche \\
\hline 6 & 92 & Frédéric & il y a/ il y a une chatte// \\
\hline
\end{tabular}

L'identification d'une information se fait également à partir d'un verbe identique ou équivalent sémantiquement au verbe du texte-source comme le montrent ces trois formulations se rapportant à l'information suivante: "Sa queue frétille d'impatience » (information 8).

Tableau 5. Exemples d'informations verbales

\begin{tabular}{|l|l|l|l|}
\hline Séances & $\mathbf{N}^{\mathbf{0}}$ & Loc & Interventions \\
\hline 8 & 78 & Tania & $\begin{array}{l}\text { euh quand/ comme il avait enlevé son gilet et et l'a vu une mare avec une } \\
\text { chatte prêt à attraper un poisson rouge elle bougeait sa queue de } \\
\text { patience et et après Pierre est tombé dans la douceur de son terrier et } \\
\text { s'effondre par terre endormi et sa maman dit/ euhm comment as-tu } \\
\text { enlevé ton gilet ça fait deux jours }\end{array}$ \\
\hline
\end{tabular}




\begin{tabular}{|l|l|l|l|}
\hline 9 & 43 & Luc & $\begin{array}{l}\text { le sat(chat) il voulait attraper un poisson sa queue frétille et/ mais } \\
\text { Pierre ne le veut pas la déranger }\end{array}$ \\
\hline 10 & 170 & Lucie & sa queue elle frétille d'impatience \\
\hline
\end{tabular}

\section{Les critères d'analyse}

L'objet de notre étude est de montrer qu'en situation collective, lorsqu'il existe un travail de collaboration, de jeunes élèves peuvent proposer une restitution de récit, dont le résultat est supérieur à celui qu'on pourrait obtenir dans un travail individuel ou en dialogue pédagogique ordinaire. Il s'agit donc de recenser au sein de chacune des séances les informations restituées, puis de les identifier, en fonction de leur importance au regard de la compréhension de l'ensemble de l'épisode, selon qu'elles sont principales ou secondaires.

40 L'analyse proprement dite des séances portera sur :

- le nombre d'informations ;

- la nature de ces informations et en particulier l'apparition des informations de second plan ;

- la verbalisation de ces informations dans le jeu collectif de la séance.

\section{Les résultats}

41 Les trois points qui suivent sont consacrés aux résultats généraux sur l'ensemble des séances analysés dans ce corpus. Ils concernent tout d'abord les données restituées, la prise en charge collective des informations et enfin l'étude comparative de deux séances afin d'éclairer les variations possibles dans le retrait de l'enseignant qui favorise ainsi plus ou moins la coopération.

\section{Les données restituées}

Le recours aux outils informatiques permet d'obtenir un aperçu sur la place et l'importance des informations rapportées par les élèves. Cette analyse doit être approfondie par une étude précise des outils de la langue mobilisés par les élèves pour rendre compte des informations retenues.

\section{Analyse quantitative}

43 Si on met en parallèle les informations et le nombre de restitutions sur l'ensemble des séances, on s'aperçoit que les restitutions des données apparaissent dans des proportions variables. Certaines informations sont mieux relayées. 
Tableau 6. Nombre total d'informations reprises sur l'ensemble des séances

\begin{tabular}{|l|l|l|l|l|l|l|l|l|l|l|l|}
\hline $\mathbf{N}^{0}$ & $\mathbf{1}$ & $\mathbf{2}$ & $\mathbf{3}$ & $\mathbf{4}$ & $\mathbf{5}$ & $\mathbf{6}$ & $\mathbf{7}$ & $\mathbf{8}$ & $\mathbf{9}$ & $\mathbf{1 0}$ & $\mathbf{1 1}$ \\
\hline Information & $\begin{array}{l}\text { Pierre } \\
\text { court }\end{array}$ & $\begin{array}{l}\text { Il veut } \\
\text { sortir du } \\
\text { potager }\end{array}$ & $\begin{array}{l}\text { Il } \\
\text { arrive } \\
\text { à la } \\
\text { mare }\end{array}$ & $\begin{array}{l}\text { Il y a } \\
\text { une } \\
\text { chatte }\end{array}$ & $\begin{array}{l}\text { Il y a un } \\
\text { poisson }\end{array}$ & $\begin{array}{l}\text { La } \\
\text { chatte } \\
\text { observe } \\
\text { le } \\
\text { poisson }\end{array}$ & $\begin{array}{l}\text { Elle veut } \\
\text { le } \\
\text { manger }\end{array}$ & $\begin{array}{l}\text { Sa } \\
\text { queue } \\
\text { frétille }\end{array}$ & $\begin{array}{l}\text { Pierre ne } \\
\text { la dérange } \\
\text { pas }\end{array}$ & $\begin{array}{l}\text { Il a un } \\
\text { doute... }\end{array}$ & $\begin{array}{l}\text { Son } \\
\text { cousin } \\
\text { l'en a } \\
\text { informé }\end{array}$ \\
\hline $\begin{array}{l}\text { Nombre de } \\
\text { restitutions sur } \\
\text { l'ensemble des } \\
\text { séances }\end{array}$ & 25 & 0 & 13 & 14 & 6 & 7 & 7 & 4 & 14 & 17 & 8 \\
\hline
\end{tabular}

Parmi elles, les informations principales - en vert dans la ligne «information » - sont majoritaires. Elles concernent trois informations de premier plan, liées à la temporalité objective du récit (Pierre court, il arrive à la mare et il ne dérange pas la chatte) et deux informations de second plan (Il y a une chatte et Pierre a un doute... il ne sait pas si les chats sont les ennemis des lapins, véritable question en monologue intérieur pour laquelle le personnage n'a pas de réponse).

\section{Analyse qualitative}

L'analyse va donc porter sur ces informations principales. La course est l'information la plus fréquemment restituée. La mémorisation de cette donnée est facilitée par le récit luimême puisque ce thème, mentionné à cinq reprises, sert de fil conducteur aux péripéties qui s'enchainent. La forme de la séquence verbale la course folle/ reprend de plus belle séduit sans doute également les enfants. Les reformulations exhibent l'intérêt des élèves pour cette expression.

Tableau 7. Intérêt des élèves pour l'expression la course folle/ reprend de plus belle

\begin{tabular}{|c|c|c|c|}
\hline Séance & $\mathrm{N}^{\circ}$ & Loc & Interventions \\
\hline 9 & 4 & Vicky & $\begin{array}{l}\text { // euh il s'est enfoui euh et monsieur Grégory il a pris un panier pour } \\
\text { attraper Pierre et Pierre il a sauté dans l'arrosoir il avait froid et il fallait } \\
\text { pas qu'il bouge monsieur Grégory le cherchait partout et heu il a fait il a } \\
\text { fait atchoum et la course folle elle reprend de sa plus belle }\end{array}$ \\
\hline 9 & 24 & Kader & $\begin{array}{l}\text { il a saut Pierre il a sauté dans l'arrosoir mais l'eau elle était froide et il a } \\
\text { fait atchoum et après il a ressauté et recourt vers sa vitesse et il voit un } \\
\text { poisson mais il veut le manger mais quand même il ose pas le déranger }\end{array}$ \\
\hline 9 & 30 & Sonia & Pierre il a fait une corse(course) une course bien belle \\
\hline 9 & 34 & Luc & et la course folle reprend de plus belle \\
\hline 9 & 42 & Amélie & $\begin{array}{l}\text { Pierre il il la course elle reprend de plus en plus belle et et il Pierre a } \\
\text { couru le ventre à terre }\end{array}$ \\
\hline
\end{tabular}

L'arrivée à la mare, nouveau lieu de référence du récit, est reformulée. Cet ancrage spatial situe cet épisode dans le thème plus vaste de la fuite: c'est donc une information principale décisive. La localisation peut être donnée de manière statique, pour situer Pierre, ou dynamique comme le résultat de sa trajectoire. 
Tableau 8. Localisation donnée de manière statique ou dynamique

\begin{tabular}{|l|l|l|l|l|}
\hline Séances & $\mathbf{N}^{\mathbf{0}}$ & Loc & Interventions & localisation \\
\hline 3 & 185 & Reda & quand::: / quand il::: / quand il trouve la mare & statique \\
\hline 3 & 214 & Marianne & et après/ il va à la mare & dynamique \\
\hline 3 & 248 & Marianne & et après/ il va dans la & dynamique \\
\hline 3 & 251 & Reda & il arrive dans la mare & dynamique \\
\hline 11 & 51 & Théophile & $\begin{array}{l}\text { et aussi il arrive près d'une mare où une chatte } \\
\text { blanche observe un poisson rouge/ sa queue frétille } \\
\text { sachant que faire/ comme lui a dit son cousin les chats } \\
\text { sont les ennemis des lapins/ il s'arrête de courir juste } \\
\text { seulement quand il est arrivé dans la douceur de son } \\
\text { terrier }\end{array}$ & dynamique \\
\hline 9 & 25 & Viviane & $\begin{array}{l}\text { il voyait une mare/ de canard et puis Pierre il aperçoit } \\
\text { un poisson et puis il dit/ j'aurai un bon diner Pierre je } \\
\text { me ferai::: }\end{array}$ & statique \\
\hline
\end{tabular}

rencontre de la chatte et la réaction de Pierre puis passe à la suite. En mêlant des informations principales et des informations secondaires elle avait un poisson pour son diner , Keziah (S7-46) résume également l'épisode de la mare, mais de manière moins cohérente que Shona. externe, mais un état interne. Les élèves reconnaissent bien l'importance de cette dernière Pierre ne sait pas si les chats sont vraiment les ennemis des lapins, qui, même si elle relève du second plan, est une information principale dans cet épisode. C'est pourquoi ils 
sont nombreux à la rapporter. Or, la reformulation peut comporter ou non une modalisation, celle du doute ou de la certitude, comme le montre la grande variété des énoncés ci-dessous.

Tableau 9. Épisode de la mare

\begin{tabular}{|c|c|c|c|}
\hline Séances & $\mathrm{N}^{\mathrm{o}}$ & Loc & Interventions \\
\hline 2 & 96 & Gaël & Pierre// et après la course folle reprend// \\
\hline 2 & 97 & Sandra & après il voit le chat? \\
\hline 2 & 111 & Sandra & $\begin{array}{l}\text { et Pierre il voit un chat il ne veut pas le déranger tout doucement il } \\
\text { s'assit et il trouve le chemin// et il rentre chez lui et il dit Pierre qu'est- } \\
\text { ce que t'as/ je te trouve un peu bizarre mais c'est la deuxième fois que } \\
\text { tu perds tes vêtements/ tiens je te donne une bonne tisane pour que tu } \\
\text { souhaites une bonne nuit// }\end{array}$ \\
\hline 6 & 24 & Emilie & $\begin{array}{l}\text { près du lac il y avait une chatte blanche et il savait pas si son cousin il } \\
\text { avait raison/ si les chats ils étaient les ennemis des lapins }\end{array}$ \\
\hline 6 & 106 & Alexis & $\begin{array}{l}\text { il y a aussi/ la chatte et puis les poissons// c'est un poisson normal et } \\
\text { la chatte elle veut l'attraper le poisson/ elle en ferait bien un dîner }\end{array}$ \\
\hline 8 & 78 & Tania & $\begin{array}{l}\text { euh quand/ comme il avait enlevé son gilet et et l'a vu une mare avec } \\
\text { une chatte prêt à attraper un poisson rouge elle bougeait sa queue de } \\
\text { patience et et après Pierre est tombé dans la douceur de son terrier et } \\
\text { s'effondre par terre endormi et sa maman dit/ euhm comment as-tu } \\
\text { enlevé ton gilet ça fait deux jours }\end{array}$ \\
\hline 1 & 33 & Shona & $\begin{array}{l}\text { eh bien il a perdu sa chaussure dans les pommes de terre et après son } \\
\text { blouson il s'est accroché au fil et après à ce moment il enlève son gilet et } \\
\text { après il rentre dans la remise et sans réfléchir il saute dans l'arrosoir et } \\
\text { il a froid car il est à moitié dans l'eau et après il éternue et après } \\
\text { monsieur Grégory qui l'avait pas vu il se retourne et après il court/ il } \\
\text { court/ il arrive près d'un champ/ il rencontre un chat/ mais il } \\
\text { n'ose pas lui demander et après Pierre quand il est arrivé chez lui/ il } \\
\text { monte dans sa maison }\end{array}$ \\
\hline 7 & 46 & Keziah & $\begin{array}{l}\text { hum Pierre/ il a fait atchoum quand il était dans l'eau froide/ monsieur } \\
\text { Grégory/ il lui a dit par mes moustaches/ je vais te transformer en pâté } \\
\text { et la chatte elle avait un poisson/ elle avait un poisson pour son } \\
\text { dîner/ Pierre a dit je vais pas te déranger/ }\end{array}$ \\
\hline
\end{tabular}

Tableau 10. Variation dans la restitution

\begin{tabular}{|l|l|l|l|l|}
\hline Séances & $\mathbf{N}^{\mathbf{0}}$ & Loc & Interventions & \\
\hline 3 & 253 & Marianne & parce qu'ils sont vraiment les ennemis des lapins & Certitude \\
\hline
\end{tabular}




\begin{tabular}{|l|l|l|l|l|}
\hline 3 & 262 & Malo & il a dit que les chats sont les ennemis des lapins & Certitude \\
\hline 4 & 86 & Judicaël & $\begin{array}{l}\text { et il passe en dessous des jambes de monsieur Grégory et } \\
\text { après il voit une chatte et il se souvient qu'est-ce que son } \\
\text { cousin lui avait dit/ les chats ce sont les ennemis des } \\
\text { lapins }\end{array}$ & Certitude \\
\hline 6 & 24 & Emilie & $\begin{array}{l}\text { près du lac il y avait une chatte blanche et il savait pas si } \\
\text { son cousin il avait raison/ si les chats ils étaient les } \\
\text { ennemis des lapins }\end{array}$ & Doute \\
\hline 11 & 51 & Théophile & $\begin{array}{l}\text { Pierre ne veut pas la déranger ne sachant que faire/ } \\
\text { comme lui a dit son cousin les chats sont les ennemis } \\
\text { des lapins/ il s'arrête de courir juste seulement quand il } \\
\text { est arrivé dans la douceur de son terrier }\end{array}$ & Doute \\
\hline 12 & 13 & Jean & $\begin{array}{l}\text { il a vu un chat et i(l) se demandait si le chat si c'était le } \\
\text { pire ennemi des lapins }\end{array}$ & Doute \\
\hline
\end{tabular}

Les élèves se rappellent que Pierre pense quelque chose mais ils ne retiennent cette pensée que comme "cause » de son choix, d'où le fait que certains élèves (Marianne, Malo, Judicaël) transforment la réflexion de Pierre en certitude alors qu'il s'agissait d'un doute. Autrement dit, en racontant ils renforcent la cohérence d'origine.

\section{La prise en charge collective des informations}

Le recueil des informations à l'intérieur de chaque séance montre une grande variété de restitution de l'épisode. Dans le tableau qui suit, nous comptabilisons le nombre d'interventions correspondant à chacune des informations constituant l'épisode dans chaque séance du corpus.

Sur le tableau, les informations principales sont respectivement en vert et les secondaires en orange.

Tableau 11. Nombre d'informations restituées par séance

\begin{tabular}{|c|c|c|c|c|c|c|c|c|c|c|c|c|c|c|}
\hline No & Information & 1 & 2 & 3 & 4 & 5 & 6 & 7 & 8 & 9 & 10 & 11 & 12 & Total \\
\hline 1 & Pierre court & 3 & 1 & 4 & 3 & 1 & 2 & & 2 & 5 & 1 & 1 & 2 & 25 \\
\hline 2 & $\begin{array}{l}\text { Il veut sortir du } \\
\text { jardin }\end{array}$ & & & & & & & & & & & & & 0 \\
\hline 3 & Il arrive à la mare & 1 & & 4 & 1 & 1 & 1 & & 1 & 1 & & 2 & 1 & 13 \\
\hline 4 & Il y a une chatte & 1 & 2 & 2 & 2 & 1 & 4 & & & & 1 & & 1 & 14 \\
\hline 5 & Il y a un poisson & & & & & & 3 & & & 2 & 1 & & & 6 \\
\hline 6 & $\begin{array}{l}\text { La chatte observe } \\
\text { le poisson }\end{array}$ & & & 1 & 1 & 2 & & 1 & 1 & & & 1 & & 7 \\
\hline 7 & $\begin{array}{l}\text { La chatte veut } \\
\text { manger le poisson }\end{array}$ & & & 1 & & 1 & 1 & 1 & & 3 & & & & 7 \\
\hline 8 & Sa queue frétille & & & & & & & & 1 & 1 & 1 & 1 & & 4 \\
\hline 9 & $\begin{array}{l}\text { Pierre ne la } \\
\text { dérange pas }\end{array}$ & 1 & 1 & 3 & 2 & 1 & & 1 & & 3 & & 1 & & 13 \\
\hline 10 & Il a un doute & & & 8 & 4 & 1 & 1 & & & 1 & & 1 & 1 & 17 \\
\hline 11 & $\begin{array}{l}\text { Son cousin le lui a } \\
\text { dit }\end{array}$ & & & 2 & 2 & 1 & 1 & & & 1 & & 1 & & 8 \\
\hline
\end{tabular}



informations principales, même s'il s'agit d'états mentaux comme les informations 9 et 10. Les informations secondaires sont globalement moitié moins nombreuses.

Le travail collectif est sensible dans les enchainements. Quand l'information 3 est donnée (Pierre arrive à la mare) alors l'information 1 est donnée (Il court). L'information 9 (Pierre ne dérange pas la chatte) et l'information 10 (Il a un doute, il ne sait pas si les chats sont vraiment les ennemis des lapins) sont dans une relation moins nette. Si la 9 est donnée plusieurs fois, alors on donne la 10 qui est cause de la 9. Lorsque l'information 11 est présente (Son cousin le lui a dit), on peut avoir des enchainements complets. Ces enchainements sont visibles en gras dans le tableau.

58 Les séances 3 et 4 étant celles qui traitent le plus longuement de l'épisode qui nous intéresse, nous allons les examiner en particulier. Notre analyse portera essentiellement sur les interactions ou les échanges entre élèves.

\section{Étude des séances}

Les deux séances n'ont pas le même style et les élèves n'interagissent pas de la même manière, ce qui nous permet de les comparer.

\section{La séance 3}

Tableau 12. Marianne et Clarissa : collaboration ou concurrence

\begin{tabular}{|c|c|c|c|c|}
\hline Séance & $\mathrm{N}^{\circ}$ & Loc & Interventions & \\
\hline 3 & 185 & Reda & $\begin{array}{l}\text { quand::: / quand il::: / quand } \\
\text { il trouve la mare }\end{array}$ & \\
\hline 3 & 186 & M & $\begin{array}{l}\text { ce n'est pas encore tout de } \\
\text { suite// mais avant d'être allé } \\
\text { dans la remise/ que lui est-il } \\
\text { arrivé }\end{array}$ & $\begin{array}{l}\text { La maîtresse propose un retour à la } \\
\text { chronologie. La gestion assez directive } \\
\text { de la restitution détermine un certain } \\
\text { type d'échanges ou d'interventions }\end{array}$ \\
\hline 3 & 213 & Clarissa & $\begin{array}{l}\text { il arrive pas à trouver le trou } \\
\text { par où il est entré }\end{array}$ & \\
\hline 3 & 214 & Marianne & et après/ il va à la mare & \\
\hline 3 & 215 & Clarissa & et après/ il a trouvé & $\begin{array}{l}\text { De } 213 \text { à } 215 \text {, les deux élèves peuvent } \\
\text { travailler ensemble ou se concurrencer }\end{array}$ \\
\hline 3 & 216 & M & $\begin{array}{l}\text { oui Sam?/ qu'est-ce qu'il est } \\
\text { arrivé }\end{array}$ & $\begin{array}{l}\text { La question assez précise ne laisse pas } \\
\text { développer l'échange au hasard des } \\
\text { réponses }\end{array}$ \\
\hline 3 & 248 & Marianne & et après/ il va dans la & \\
\hline 3 & 249 & El(s) & il va dans la mare & \\
\hline
\end{tabular}




\begin{tabular}{|l|l|l|l|l|}
\hline 3 & 250 & Clarissa & $\begin{array}{l}\text { une chatte blanche/ une } \\
\text { chatte blanche }\end{array}$ & $\begin{array}{l}\text { La collaboration se poursuit mais avec } \\
\text { des interventions très courtes, comme } \\
\text { s'il fallait être la première à donner } \\
\text { l'information }\end{array}$ \\
\hline 3 & 251 & Reda & il arrive dans la mare & $\begin{array}{l}\text { Reda calme le jeu en reformulant 249, } \\
\text { auquel il a peut-être participé }\end{array}$ \\
\hline 3 & 252 & Clarissa & $\begin{array}{l}\text { poisson rouge dont elle ferait } \\
\text { son repas et Pierre il veut pas } \\
\text { la déranger }\end{array}$ & $\begin{array}{l}\text { Clarissa enchaine sur elle-même pour } \\
\text { une plus longue intervention. }\end{array}$ \\
\hline 3 & 253 & Marianne & $\begin{array}{l}\text { parce qu'ils sont vraiment les } \\
\text { ennemis des lapins }\end{array}$ & $\begin{array}{l}\text { Marianne à sa suite enchaîne } \\
\text { directement. }\end{array}$ \\
\hline 3 & 254 & Reda & $\begin{array}{l}\text { son cousin il a dit que c'est } \\
\text { l'ennemi des lapins }\end{array}$ & $\begin{array}{l}\text { Reda reformule une nouvelle fois ce qui } \\
\text { a déjà été présenté }\end{array}$ \\
\hline
\end{tabular}

Tableau 13. De la difficulté de collaborer

\begin{tabular}{|c|c|c|c|c|}
\hline Séance & $\mathrm{N}^{\mathrm{o}}$ & Loc & Interventions & \\
\hline 3 & 255 & $M$ & $\begin{array}{l}\text { donc qu'est-ce qu'il } \\
\text { fait quand il voit la } \\
\text { chatte }\end{array}$ & $\begin{array}{l}\text { L'enseignant reprend la main: il ne s'appuie pas } \\
\text { sur ce que viennent de dire les élèves, il } \\
\text { remonte en amont dans la restitution de } \\
\text { l'épisode }\end{array}$ \\
\hline 3 & 256 & Viviane & $\begin{array}{l}\text { il n'ose pas la } \\
\text { déranger }\end{array}$ & $\begin{array}{l}\text { Une autre élève intervient. La question de } \\
\text { l'enseignant a rendu possible son intervention. }\end{array}$ \\
\hline 3 & 257 & $M$ & $\begin{array}{l}\text { qu'est-ce qu'il fait/ } \\
\text { Alex }\end{array}$ & Sollicitation directe et individualisée. \\
\hline 3 & 258 & Melwan & $\begin{array}{l}\text { il fait la pointe des } \\
\text { pieds pour qu'il la } \\
\text { voye }\end{array}$ & $\begin{array}{l}\text { Reformulation de la séquence du texte qui est } \\
\text { bien comprise. }\end{array}$ \\
\hline 3 & 259 & Marianne & $\begin{array}{l}\text { parce qu'ils sont les } \\
\text { ennemis des lapins }\end{array}$ & $\begin{array}{l}\text { Marianne reprend son propos (253) et justifie la } \\
\text { réponse de Melwan en s'octroyant une place } \\
\text { dans l'interaction. }\end{array}$ \\
\hline 3 & 260 & Clarissa & peut-être & $\begin{array}{l}\text { Clarissa intervient à la suite. Ce "peut-être" est } \\
\text { ouvert à plusieurs interprétations.... }\end{array}$ \\
\hline 3 & 261 & $M$ & $\begin{array}{l}\text { peut-être/ } \\
\text { exactement }\end{array}$ & $\begin{array}{l}\text { L'enseignant le réfère uniquement au contenu. } \\
\text {.. }\end{array}$ \\
\hline
\end{tabular}




\begin{tabular}{|l|l|l|l|l|}
\hline 3 & 262 & Malo & $\begin{array}{l}\text { il a dit que les chats } \\
\text { sont les ennemis des } \\
\text { lapins }\end{array}$ & $\begin{array}{l}\text { Malo propose une nouvelle intervention, mais } \\
\text { qui reste difficile à interpréter. Qui est « il »? } \\
\text { Est-ce une remise en cause de Marianne (il = } \\
\text { elle) ? Est-ce une référence au cousin de Pierre? }\end{array}$ \\
\hline
\end{tabular}

Le couple de Marianne et Clarissa fonctionne toujours dans ce passage, de manière oppositive ou concurrentielle. Cela pose évidemment problème aux autres élèves qui doivent s'insérer dans ce duel verbal. Les interventions de Melwan et Malo illustrent cette difficulté.

\section{Tableau 14. De la difficulté d'une gestion directive}

\begin{tabular}{|l|l|l|l|l|}
\hline Séance & $\mathbf{N}^{\mathbf{0}}$ & Loc & Interventions & \\
\hline 3 & 263 & M & $\begin{array}{l}\text { comment sait-il que le } \\
\text { chat peut être } \\
\text { dangereux }\end{array}$ & $\begin{array}{l}\text { L'enseignant oriente les élèves sur une } \\
\text { information secondaire et cela va être un } \\
\text { échec }\end{array}$ \\
\hline 3 & 264 & Viviane & parce qu'il a des griffes & \\
\hline 3 & 265 & Clarissa & $\begin{array}{l}\text { il sort ses griffes } \\
\text { comme danger/ il y } \\
\text { avait d'abord monsieur } \\
\text { Grégory et après la la } \\
\text { chatte }\end{array}$ & $\begin{array}{l}\text { l'une certaine façon Reda le reformulateur est } \\
\text { une intervention qu'on pourrait qualifier de } \\
\text { raisonnable par rapport au thème donné par } \\
\text { l'enseignant. }\end{array}$ \\
\hline 3 & 266 & Reda prise de recul qui lui permet ici \\
\hline Clarissa & $\begin{array}{l}\text { monsieur Grégory/ça va } \\
\text { faire beaucoup pour } \\
\text { Pierre a la chatte et le }\end{array}$ & \\
\hline
\end{tabular}

61 Le tableau synthétise les informations données lors de cette restitution ainsi que les formulations utilisées par les élèves. 
Tableau 15. Synthèse

\begin{tabular}{|c|c|c|c|c|}
\hline Séquence verbale & $\mathbf{N}^{o}$ & $\begin{array}{l}\text { Information } \\
\text { de premier plan }\end{array}$ & $\begin{array}{l}\text { Information } \\
\text { de deuxième plan }\end{array}$ & Restitution \\
\hline $\begin{array}{l}\text { La course folle reprend de plus } \\
\text { belle }\end{array}$ & 1 & $\begin{array}{l}\text { Pierre court, et court encore } \\
\text { plus vite }\end{array}$ & & $\begin{array}{l}\text { il arrive pas à trouver le trou } \\
\text { par où il est entré }\end{array}$ \\
\hline $\begin{array}{l}\text { Il cherche désespérément à sortir } \\
\text { du potager }\end{array}$ & 2 & & Il veut sortir du potager. & \\
\hline Il arrive près d'une mare & 3 & Pierre arrive à la mare. & & $\begin{array}{l}\text { et après/ il va dans la / il va } \\
\text { dans la mare / et après/ il va } \\
\text { à la mare }\end{array}$ \\
\hline \multirow[t]{3}{*}{$\begin{array}{l}\text { Où une chatte blanche observe un } \\
\text { poisson rouge }\end{array}$} & 4 & & Il y a une chatte blanche. & $\begin{array}{l}\text { elle avait trouvé un gros } \\
\text { poisson rouge dont elle ferait } \\
\text { son repas }\end{array}$ \\
\hline & 5 & & Il y a un poisson rouge. & \\
\hline & 6 & & La chatte observe un poisson. & \\
\hline Dont elle ferait bien son repas. & 7 & & $\begin{array}{l}\text { La chatte veut manger le } \\
\text { poisson. }\end{array}$ & $\begin{array}{l}\text { elle avait trouvé un gros } \\
\text { poisson rouge dont elle ferait } \\
\text { son repas }\end{array}$ \\
\hline Sa queue frétille d'impatience & 8 & . & Sa queue frétille. & \\
\hline Et Pierre n'ose pas la déranger & 9 & $\begin{array}{l}\text { Pierre ne dérange pas la } \\
\text { chatte. }\end{array}$ & & $\begin{array}{l}\text { il fait la pointe des pieds } \\
\text { pour qu'il la voye (pas) / il } \\
\text { n'ose pas la déranger / et } \\
\text { Pierre il veut pas la déranger }\end{array}$ \\
\hline $\begin{array}{l}\text { Ne sachant pas si les chats sont les } \\
\text { ennemis des lapins }\end{array}$ & 10 & & $\begin{array}{l}\text { Pierre a un doute... il ne sait } \\
\text { pas si les chats sont les } \\
\text { ennemis des lapins. }\end{array}$ & $\begin{array}{l}\text { il a dit que les chats sont les } \\
\text { ennemis des lapins il arrive } \\
\text { dans la mare / parce qu'ils } \\
\text { sont vraiment les ennemis } \\
\text { des lapins / son cousin il a dit } \\
\text { que c'est l'ennemi des lapins } \\
\text { / peut-être! }\end{array}$ \\
\hline Comme le lui a dit son cousin & 11 & & $\begin{array}{l}\text { Le cousin de Pierre a donné } \\
\text { une information. }\end{array}$ & son cousin il a dit \\
\hline
\end{tabular}

62 Les informations principales sont toutes données, mais des informations complémentaires ont été également ajoutées à cet épisode : le chat est dangereux « parce qu'il a des griffes » et donc « il sort ses griffes ». Certains élèves comptabilisent également les sources de danger pour Pierre « comme danger/ il y avait d'abord monsieur Grégory et après la chatte ", ce qui permet alors de commenter "s'il a la chatte et le monsieur Grégory/ça va faire beaucoup pour Pierre ».

63 Finalement, les informations principales ont été redonnées, mais on ne peut pas s'empêcher d'éprouver un certain malaise. Les interactions entre élèves sont loin de relever de la collaboration. Les compétences des élèves sont celles d'une section de moyens en éducation prioritaire.

\section{La séance 4}

64 La séance 4 présente une unité thématique plus importante, dans la mesure où les élèves restituent les différentes informations dans l'ordre chronologique. Ils ne sont donc pas interrompus par l'enseignant.

65 Judicaël est d'abord le maitre d'œuvre de la restitution, il intervient 4 fois, c'est lui qui fait l'intervention la plus longue (86). Mélissa (92 et suivantes) va jouer le même rôle dans la deuxième partie de la séquence découpée ici. Entre les deux, une intervention définitoire de Naomi va déclencher un échange commentatif sur les chats.

Tableau 16. Restitution de l'épisode extrait de la séance 4

\begin{tabular}{|l|l|l|l|l|}
\hline Séance & $\mathrm{N}^{\mathrm{o}}$ & Loc & Interventions & \\
\hline
\end{tabular}




\begin{tabular}{|c|c|c|c|c|}
\hline 4 & 82 & M & Paulo & \\
\hline 4 & 83 & Paulo & $\begin{array}{l}\text { monsieur Grégory se retourne vers lui avec } \\
\text { un panier pour l'attraper }\end{array}$ & \\
\hline 4 & 84 & Judicaël & et après & \\
\hline 4 & 85 & M & oui/ Judicaël & \\
\hline 4 & 86 & Judicaël & $\begin{array}{l}\text { et il passe en dessous des jambes de } \\
\text { monsieur Grégory et après il voit une } \\
\text { chatte et il se souvient qu'est-ce que son } \\
\text { cousin lui avait dit/ les chats ce sont les } \\
\text { ennemis des lapins }\end{array}$ & \\
\hline 4 & 87 & Naomi & ça veut dire qu'ils sont pas amis & $\begin{array}{l}\text { Commentaire } \\
\text { «didactique " ou } \\
\text { métadiscursif de Naomi }\end{array}$ \\
\hline 4 & 88 & Mélissa & $\begin{array}{l}\text { ils sont pas amis/ ce sont les ennemis des } \\
\text { lapins }\end{array}$ & Idem de Melissa \\
\hline 4 & 89 & Judicaël & ils sont méchants & $\begin{array}{l}\text { S'engage un dialogue sur } \\
\text { les chats en général, ce } \\
\text { générique a été amorcé par } \\
\text { Naomi en } 24\end{array}$ \\
\hline 4 & 90 & Mélissa & sinon il le mord & \\
\hline 4 & 91 & Judicaël & il le mange & \\
\hline 4 & 92 & Mélissa & il a pas fait de bruit et & $\begin{array}{l}\text { Mélissa reprend la } \\
\text { restitution là où Judicaël } \\
\text { l'arrêtait en } 86 .\end{array}$ \\
\hline 4 & 93 & M & Mélissa & L'enseignant soutient. \\
\hline 4 & 94 & Mélissa & $\begin{array}{l}\text { il a regardé/ il a pas fait de bruit pour pas le } \\
\text { déranger// il est parti il a couru et après il } \\
\text { a été dans sa }\end{array}$ & $\begin{array}{l}\text { C'est bien cela, elle } \\
\text { poursuit le récit. }\end{array}$ \\
\hline 4 & 95 & M & il a été où & \\
\hline 4 & 96 & Judicaël & dans son terrier & Aide lexicale. \\
\hline 4 & 97 & Mélissa & dans son terrier et/ & \\
\hline
\end{tabular}

Le passage est certainement restitué de manière moins complète que lors de la séance trois, mais cela est fait de manière moins directive de la part de l'enseignant. Il est assez intéressant de noter que Judicaël et Mélissa occupent une place conversationnelle à peu près équivalente dans la séance, tous deux comptent 29 interventions (chacun) et 
produisent 230 mots pour l'un (longueur moyenne 8 mots) et 290 mots pour elle (longueur moyenne 10 mots).

S'il fallait parler de collaboration c'est plutôt ici qu'il faudrait mobiliser ce terme, l'absence de directive notionnelle de la part de l'enseignant, la centration de plusieurs élèves successifs sur les thèmes abordés, les phénomènes d'enchainement syntaxique, sans tension, permettent de dire cela.

\section{Conclusion}

La restitution collective d'un récit à l'école maternelle est une situation difficile pour un enseignant, chargé de "faire avancer » la narration sans délaisser une partie des élèves au profit de ceux qui auraient "la réponse attendue». C'est aussi une activité difficile pour les élèves : à quelles conditions vont-ils le faire?

Le corpus étudié répond à cette question en montrant que les élèves peuvent développer un épisode complexe sans être dirigés de manière précise par l'enseignant. La gestion même de la situation collective détermine les modalités de prise de parole. En adoptant une posture de retrait l'enseignant favorise les interactions entre les élèves. La collaboration entre en jeu lorsque les élèves interagissent pour répondre à une tâche commune et atteindre un but commun : rappeler un épisode, même peu évènementiel, dans une situation où ils prennent la parole à tour de rôle.

La restitution d'un épisode difficile pose de nombreux problèmes aux élèves : saisir les informations, les mémoriser, les reformuler, les articuler entre elles. Toutes les informations ne sont pas rappelées et la restitution de l'épisode n'est pas entièrement fidèle au texte-source. Cependant, les informations principales sont toujours restituées, en particulier celles qui appartiennent au second plan. Les erreurs commises sur le texte répondent finalement à une recherche de cohérence qui montre que les élèves réinterprètent ce qu'ils entendent de manière logique. Les échanges favorisent ces résultats, mais selon deux styles différents. Moins la prise de parole est contrainte, plus la restitution collective insère des informations principales de second plan dans la trame évènementielle.

\section{BIBLIOGRAPHIE}

BARTHES, R. (1966). «Introduction à l'analyse structurale des récits ». Communications 8, p. 1-27.

FAYOL, M. (1994). Le Récit et sa construction. Une approche de psychologie cognitive. Lausanne :

Delachaux \& Niestlé.

FRANÇOIS, F. (1989). « Langage et pensée : dialogue et mouvement discursif chez Vygotski et Bakhtine ». Enfance 42, p. 39-47. En ligne : http://www.persee.fr/web/revues/home/prescript/ article/enfan_0013-7545_1989_num_42_1_1877. 
HICKMANN, M. (2000). « Le développement de l'organisation discursive ». In : Kail, M. \& Fayol, M. (dirs), L'Acquisition du langage. Le langage en développement, au-delà de trois ans. Paris : Presses universitaires de France, p. 83-115.

JOULAIN, M. (1990). «Conversation maîtresse-enfant(s) en maternelle : la circulation de la parole ». Revue française de pédagogie 91, p. 59-67. En ligne : https://www.persee.fr/doc/ rfp_0556-7807_1990_num_91_1_1388.

MAKDISSI, H. \& BOISCLAIR, A. (2004). «Le développement de la structuration du récit chez l'enfant d'âge préscolaire ». Actes du $9^{e}$ colloque de l'AIRDF. Québec, 26-28 août. En ligne : http:// www.colloqueairdf.fse.ulaval.ca/fichier/Communications/makdissi-boisclair.pdf.

MANDLER, J.-M. \& JOHNSON, N. S. (1984). « À la recherche du conte perdu : structure de récit et rappel ». In : Denhière, G. (dir.), Il était une fois... Compréhension et souvenir de récits. Villeneuve d'Ascq : Presses universitaires du Septentrion, p. 185-230.

MINISTÈRE DE L'ÉDUCATION NATIONALE (2011). L'École maternelle, rapport nº 2011-108 de l'Inspection générale de l'Éducation nationale (V. Bouysse, P. Claus et C. Szymankiewicz, rapporteurs).

NONNON, E. (1997). « Quels outils se donner pour lire la dynamique des interactions et le travail sur les contenus de discours? ». Enjeux 39-40, p. 12-49.

PÉROZ, P. (2010). Apprentissage du langage oral à l'école maternelle. Pour une pédagogie de l'écoute. Metz : CRDP de Lorraine.

RIVIÈRE, V. (2006). L'activité de prescription en contexte didactique. Thèse en didactique des langues et des cultures : Paris III - Sorbonne nouvelle. En ligne : https://tel.archives-ouvertes.fr/ tel-00374551.

VEnEZIANo, E. \& hUdelot, C. (2005). «Conduites explicatives dans la narration et l'effet de l'étayage : méthodes d'analyse et quelques résultats quantitatifs tirés d'une étude développementale et comparative d'enfants typiques et d'enfants dysphasiques ». Travaux neuchâtelois de linguistique 42, p. 81-103.

\section{NOTES}

1. Dans cette étude, l'expérimentateur lit l'histoire de Benjamin et la nuit à l'ensemble des enfants. Par la suite, chaque enfant produit une narration individuelle à partir de la consigne «Peux-tu me raconter qu'est ce que c'est que cette histoire-là? ». Le rappel se fait livre fermé, sans aucune intervention structurante de l'adulte.

\section{RÉSUMÉS}

L'étude du rappel de récit individuel a donné lieu à de nombreuses publications, qui, toutes, pointent les difficultés de jeunes enfants à construire une narration organisée et cohérente. En situation scolaire collective, comment les enfants d'école maternelle restituent-ils un conte? En posant que les narrations enfantines sont d'abord des squelettes évènementiels, l'apparition des 
éléments du deuxième plan dans la restitution de récit est un facteur de complexification qui mérite notre attention. Nous examinons cette hypothèse à travers l'étude de la restitution d'un épisode de Danger dans le potager sur le corpus proposé. L'analyse porte d'abord sur le textesource, support de la restitution : il s'agit de la dernière étape à la mare dans la fuite de Pierre. Après avoir spécifié l'originalité de cette péripétie au sein de la course-poursuite, les informations sont identifiées au regard des séquences verbales. Elles sont ensuite classées selon les plans du récit d'une part, et selon leur importance en réception d'autre part. Un examen des relations entre les informations complète cette analyse. Le cadre de description permet d'élaborer une méthodologie basée sur le séquençage des énoncés des élèves, pour recenser les informations restituées et les identifier en fonction de leur importance. Une première approche quantitative des résultats montre que toutes les informations principales, qu'elles appartiennent au premier plan ou au deuxième plan, sont majoritaires sur l'ensemble des séances. Une analyse plus qualitative révèle la grande variété des reformulations pour une même information. Le travail collectif est ensuite examiné séance par séance: il confirme que les informations principales sont les plus rapportées, y compris celles appartenant au second plan. Il permet aussi de dégager quelques enchainements, véritables traces de ce travail de groupe. Un retour aux transcriptions de deux séances permet de constater les différences énonciatives liées aux effets de la gestion du groupe en fonction des enseignants. En conclusion, la restitution collective d'un épisode difficile est favorisée par une gestion qui autorise la collaboration des élèves. Les informations principales de second plan sont d'autant plus aisément introduites et reformulées que la parole circule entre les élèves.

The study of the individual narrative recall has led to numerous publications, all of which focus on the difficulties that young children meet when they want to structure an organized and coherent story. In a collective school context, how do children in nursery section tell a story? By posing that children's stories are first and foremost event outlines, the appearance of the second place elements is a complicating factor that deserves our attention. It is is necessary to consider this hypothesis by studying a reconsruction of an episode of Danger in the garden on the proposed corpus. The analysis focuses first of all on the original text which is the material for reconstruction: it deals with the last scene taking place at the pond during Pierre's escape. Once the original aspect of this adventure within the chase has been pointed out, the information is identified with regard to verbal sequences. They are then classified according to the story outlines on the one hand and on their importance in reception on the other hand. This analysis is completed by a study of the relationships between the information. The description framework makes it possible to develop a methodology based on the sequencing of the pupils' statements in order to collect the reconstructed information and identify it according to its importance. A first quantitative approach to the results shows that all the main information, whether it belongs to the first place or to the second place, are essential for all the lessons. A better qualitative analysis shows the large variety of reformulations for the same information. The common work is then examined lesson by lesson: it is confirmed that the main information is the most reported, including the information from the second place. It also identifies some sequences which are real traces left by this common work. It seems that a return to the transcripts of two lessons reveals the enunciative differences related to the effects of group management depending on the teachers. To conclude, the common reconstruction of a difficult episode is suported by a class management based on the joint work of pupils. The more speech flows between students, the easier it is to introduce and reformulate background information. 
INDEX

Mots-clés : restitution collective, plans du récit, gestion du groupe-classe

Keywords : common reconstruction, story outline, class management

\section{AUTEUR}

MARIANNE DRUSCH

ESPÉ de Lorraine, F-57000, France 